\title{
Digital Tracking Cloud Distributed Architecture for Detection of Faint NEAs
}

\author{
Roxana E. Sichitiu (Avram), Marc E. Frincu \\ Computer Science Department \\ West University of Timisoara \\ Timisoara, Romania \\ Email: \{roxana.sichitiu87, marc.frincu\}@e-uvt.ro
}

\author{
Ovidiu Vaduvescu \\ Astronomy department \\ Isaac Newton Group \\ La Palma, Spain, \\ School of Doctoral Sciences, \\ University of Craiova, Romania. \\ Email: ovidiu.vaduvescu@gmail.com
}

\begin{abstract}
There is an exponential volume of captured images, millions of captures taken every night being processed and scrutinized. Big Data analysis has become essential for the study of the solar system, discovery and orbital knowledge of the asteroids. This analysis often requires more advanced algorithms capable of processing the available data and solve the essential problems in almost real time. One such problem that needs very rapid investigation involves the detection of Near Earth Asteroids (NEAs) and their orbit refinement which should answer the question "will the Earth collide in the future with any hazardous asteroid?". This paper proposes a cloud distributed architecture meant to render near real-time results, focusing on the image stacking techniques aimed to detect very faint moving objects, and pairing of unknown objects with known orbits for asteroid discovery and identification.
\end{abstract}

Index Terms-Image Processing; Cloud computing; Distributed Systems; Big Data; Data Mining analysis; Stacking; Orbit Pairing; NEA;

\section{INTRODUCTION}

Mankind has been attracted by the sky since its beginings, and astronomy has been studied since the earliest centuries. In the past couple of decades the information collected by ground, air and space instruments increased exponentially in comparison with the 20th century. The last five decades have witnessed a boom regarding the capacity to store the information, as well as the ease of accessing it in a distributed fashion. The information started by being kept on physical disks, but later it slowly migrated to a new concept of being processed and stored, namely cloud computing [1]. The offer of cloud solutions has an ascending trend due to the optimization of data losses, economic advantage, accessibility, and also processing power. [1].

Cloud computing is a very handy solution applicable in multiple domains and astronomy is one of them. Proved by some unfortunate asteroids collisions with the Earth (the most recent asteroid that impacted Earth in 2015 was $20 \mathrm{~m}$ in size (!), leading to over 2,000 wounded victims in Chelyabinsk, Russia), the USA government mandated NASA to discover by 2030 all NEAs larger than $100 \mathrm{~m}$ and to classify their path. Some of these bodies are defined as "virtual impactors" (VIs) (referring to a set of about 1000 known NEAs which have a slim but possible chance to impact the Earth in the future according to the current poor knowledge about their orbits). The classification of an orbit defining such VIs involves a varying observing coverage time, starting from a few days to a few weeks upon discovery of each object.

Storing and processing this data on clouds is a natural approach, however, most existing tools were not designed with parallel and distributed capabilities (cf. Section III. The collected information requires intelligent software pipelines to process very rapidly the big amount of images, and to scale large data volumes. There are more than one million tracks (unknown objects observed during only one night) in need of pairing with more than 800,000 known asteroids - which requires a great calculation power and storage as detailed below (see also Eq. (1)).

A cloud distributed solution that would scale well for VIs and other NEAs regardless the type of infrastructure is needed. A reason for investing in such a solution would be that the computation is made closer to the geographical source, leading to saving money in case of big data processing. Even thought there are some distributed legacy systems (e.g. Condor) that are already doing a great job, the advantage that cloud brings is the pay-per-use system. This payment system is adaptable to the user needs, the payment will decrease/increase depending of the utilized resources.

The objective of this paper is to propose a distributed cloud enabled architecture for one modern technique used in discovery of faint NEAs, namely digital tracking. Compared with the classical "blink" method, digital tracking has similar receipes to implement, up to a point:

- Taking multiple consecutive images of one star field;

- Processing the images of background noise and optical distorsions;

- Aligning the images;

From this point each technique proceeds as follows:

- For digital tracking, analyze all possible directions of an unknown asteroid by:

- Choosing one arbitrary starting point (image) for tracking;

- Choosing a random direction and speed for the tracking of images refered to the starting point; 


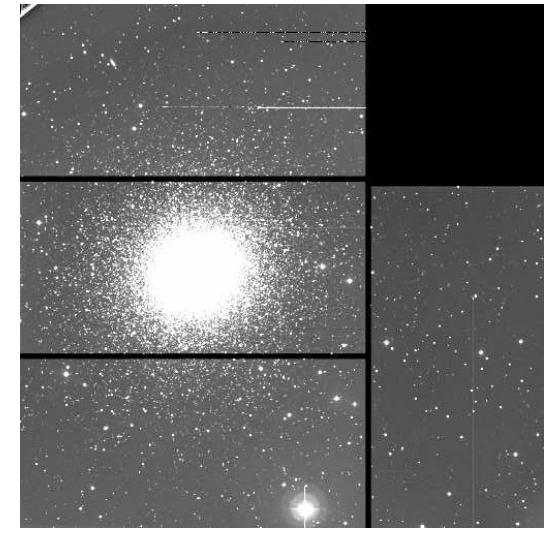

Fig. 1: Isaac Newton Telescope (INT) - Wide Field Camera (WFC) with 4 CCDs available to make the capture.

- Stacking the resulted images to increase signal to noise $(\mathrm{S} / \mathrm{N})$ needed to discover faint NEA invisible in the individual images.

- For the classic blink method (implemented elsewhere):

- Identifying asteroids in individual reduced images which appear to move linearily against aligned fixed stars;

- Compare the asteroid positions with all known asteroids for the identification of matches with known objects.

These techniques will be detailed in Section II.

There are a series of classic professional astronomy tools like IRAF and SExtractor used for cleaning up and reducing the images inputed as pixel array form in the FITS format, respectively building a catalogue of objects (detections) from the reduced astronomical images. If image acquisition cadence is fast, the example below shows that running these image reduction tools in a sequential manner can take a lot of time (up to days). By the time the final information is rendered, some of the potential asteroids might be lost or discovered by competing surveys. For capturing images, different cameras and types of telescopes are used. Let us assume for example the Isaac Newton Telescope (INT $2.5 \mathrm{~m}$ diameter) for capturing images. Its Wide Field Camera (WFC) has 4 nearby CCDs or independent "visual fields" forming a larger mosaic covering 34x34 arcmin field fitting the whole Moon (see Figure 1), which for the use case image clearing and reduction are running in a sequential manner. Assuming 100 images are taken in one field and $5 \mathrm{~min}$ for reduction of one CCD, this translates in:

100 imgs $\times 4$ CCDs/img

$$
\times 5 \mathrm{~min} / \mathrm{CCD}
$$

$$
=2,000 \mathrm{~min}
$$

for cleaning and reducing 400 images. Obviously, executing this analysis in parallel would improve performance.

The rest of the paper is structured as follows:

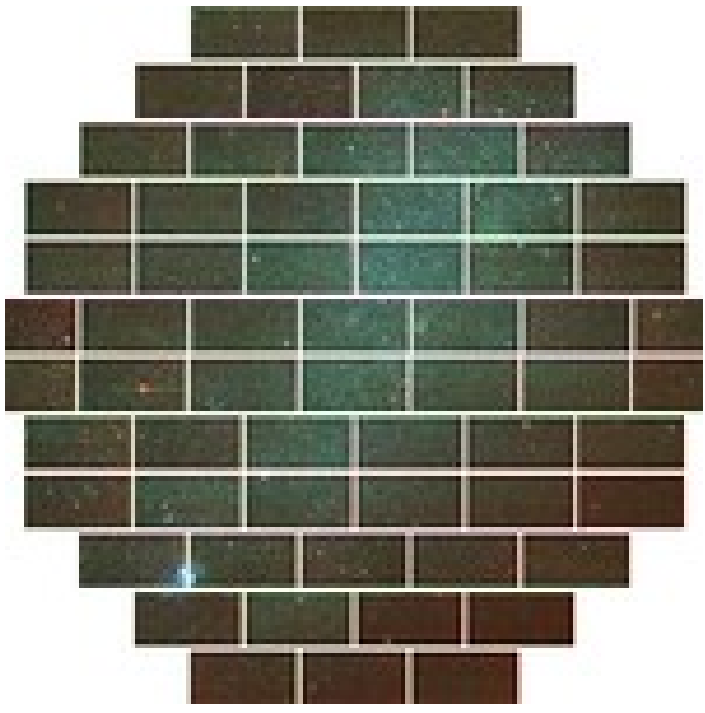

Fig. 2: Blanco telescope - DECam camera has 64 mosaic CCDs available to make the capture.
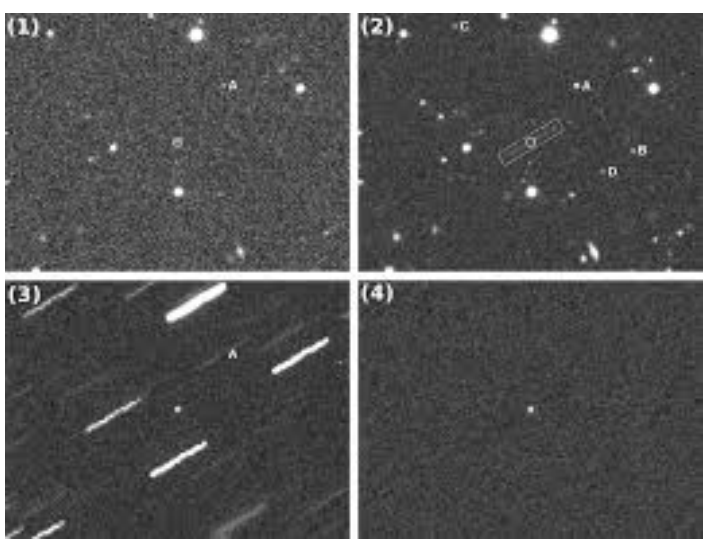

Fig. 3: Stack and track technique. [2]

1) Section II gives a brief overview on the complexity of the problem;

2) Section III makes an incursion in the domain papers and discoveries;

3) Section IV explains the proposed architecture;

4) Section IV discusses about future perspectives;

5) Section V concludes with the conclusion.

\section{Digital TRACKING - A MOTIVATING EXAMPLE}

We now give some insights on the digital tracking we consider for our study, focusing on its complexity. The idea behind this technique is to identify asteroids invisible in individual images. As a result, details need to be enhanced by tracking and stacking sequentially the reduced images, based on all possible trajectories of any unknown candidate asteroid.

A crucial step part of the digital tracking is stacking the images and tracking the potential asteroids. Figure (3) outlines its main workflow. Subfigure (1) is based on two main exposures used for finding previously unknown asteroid (circled in 
the center of the image) and a fainter Star A. Subfigure (2) represents 22 minutes of exposure that leads to blurred objects, except for the asteroid (with a trajectory), Star A and some other fainter stars. Subfigure (3) is the result of stacking and shifting 20 images that leads to finding the asteroid (bright and clear), compared to Star A and other objects. Finally, subfigure (4) contains additional cleaning of the stacked image, leaving only the found asteroid [2].

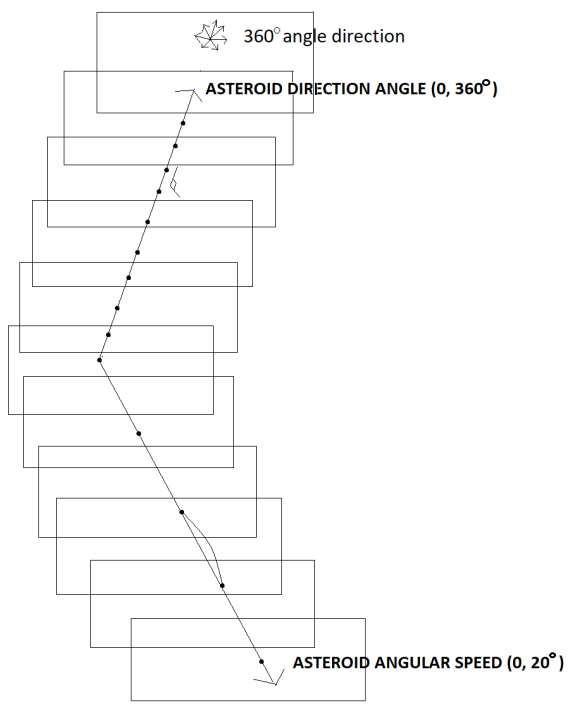

Fig. 4: Digital tracking.

For the "digital tracking" technique, "stack and track" (see Figure 4 [2]) has two different options to choose from: starting either with the direction (position angle) or the speed (proper motion), or considering at once both direction and speed.

If we chose only the position angle - we assume that NEAs can move in any direction and a step of $1 \mathrm{deg}$ is sufficient to cover all possibilities - we end up with 360 possible directions. If we instead consider only the speed, assuming a maximum speed of $20 \mathrm{arcsec} / \mathrm{min}$, a $0-20$ interval should be used. For a step of $0.1 \mathrm{arcsec} / \mathrm{min}$, we obtain 200 possible combinations.

Furthermore, if considering combining both speed and position angle for each field we would obtain:

360 directions $\times 200$ speed choiches

$$
=72,000 \text { cases }
$$

As seen in Eqs. 1 and 2 using a sequential approach can avoid the waste of time, as long as each case (tracking combination) can be executed in parallel. This is why in present conditions (when over 7 millions images need to be processed, not to mention that this type of work is made manually and visually by a group of people), new approaches are needed to process faster the accumulated Big Data. Therefore, this paper aims to propose a cloud distributed architecture able to identify faint asteroids in astronomical images, but also to scale accurately and rapidly the huge and growing amount of Big Data related to Solar system surveys, following the footsteps of the EURONEAR project (www.euronear.org) which includes 17 nodes in 9 countries [3].

\section{RELATED WORK}

Discovering NEAs typically require relatively small telescopes (1-2m diameter) and preferably very large mosaic (cf. Figure 2) cameras (few square degrees), whose operational costs are extremely high (few thousands of Euros each night), while the observing time at larger telescopes is very scarce due to other more competing science. Some objects could be accidentally recovered by the existing NEA surveys (from which Pan-STARRS, Catalina and especially the future LSST are the most competitive), but others could remain invisible for surveys due to external factors (such as faintness at future Earth approach geometry circumstances, Moon light, Milky Way, bad weather), and could remain undetected for years, while their uncertainty becomes huge and could become lost.

Only few methods exist for investigating the mitigation and prevention or deflection of the asteroids impact. In order for an object to be deflected, its orbit has to be accurately known.

One automatic solution that optimize the search in classic blink surveys was implemented in the EURONEAR NEARBY project, but in order for the search to accommodate millions of images in a fast manner necessarily distributed and scalable solutions must be used. For example, the Large Synoptic Survey Telescope (LSST) will provide a huge amount of data flowing daily, and a cloud distributed solution must be the answer for daily data reduction (as some of the previous results in literature attempted to achieve).

In the literature there are a series of efforts made in identifying new asteroids or (p)recovering the existing ones, mechanisms by which the orbit of the asteroids intended to be improved. The Table I reflect the two big sections identified in the literature: one regarding the data mining and the other - a very new area - regarding cloud based solutions.

Data mining domain in astrophysics started to be investigated almost three decades ago, in 1982 [4], but the field was insufficiently exploited and has great potential, especially in the age of larger CCD cameras.

Next, we will make a summary review regarding two classic environments of survey image processing and their capabilities so far.

The two tools are similar: the classic Image Reduction Analysis Facility (IRAF) [15] and the modern PyRAF, which is a command language for running IRAF tasks based on the Python scripting language that brings more flexibility to development [16].

PyCDA (Is an open-source library for automated crater detection)[17], followed by SExtractor or Source-Extractor, which is a program oriented towards image reduction, that builds a catalogue of objects from an astronomical image [18]. 
TABLE I: Related work overview.

\begin{tabular}{|c|c|c|c|c|c|}
\hline Paper & Type & Precov & Recov & Aim & Results \\
\hline [5] & $\begin{array}{l}\text { Data } \\
\text { mining }\end{array}$ & $\checkmark$ & $x$ & $\begin{array}{l}\text { Improving orbital infor- } \\
\text { mation and predicting dy- } \\
\text { namical evolution of NEA }\end{array}$ & 70 NEAs found \\
\hline [6] & $\begin{array}{l}\text { Data } \\
\text { mining } \\
\text { software }\end{array}$ & $\checkmark$ & $x$ & $\begin{array}{l}\text { Recover lost obj and im- } \\
\text { proving trajectories }\end{array}$ & $\begin{array}{l}13.000 \text { photographic } \\
\text { studied plates had } \\
\text { inssuficient apperture; } \\
\text { no NEAs found }\end{array}$ \\
\hline [7] & $\begin{array}{l}\text { Data } \\
\text { mining } \\
\text { archive }\end{array}$ & $\checkmark$ & $\checkmark$ & $\begin{array}{l}\text { Obtain relevant data about } \\
\text { asteroids and comets that } \\
\text { can further lead to orbit } \\
\text { improvement. }\end{array}$ & $\begin{array}{l}\text { Some asteroids were } \\
\text { found }\end{array}$ \\
\hline [8] & $\begin{array}{l}\text { Data } \\
\text { mining } \\
\text { archive }\end{array}$ & $\checkmark$ & $\checkmark$ & $\begin{array}{l}\text { Suprime-Cam captures } \\
\text { made with the Subaru } 8 \mathrm{~m} \\
\text { telescope descovered } 15 \% \\
\text { as being NEAs }\end{array}$ & $\begin{array}{l}18 \text { NEAs descovered } \\
\text { using } 2 \text { sky directons }\end{array}$ \\
\hline [2] & $\begin{array}{l}\text { Data } \\
\text { mining }\end{array}$ & $\checkmark$ & $\checkmark$ & $\begin{array}{l}\text { Uses one-degree imager } \\
\text { on the } 0.9 \mathrm{~m} \text { WIYN, ob- } \\
\text { serving into a single field. }\end{array}$ & 215 detected asteroids \\
\hline [9] & $\begin{array}{l}\text { Distributed } \\
\text { cloud } \\
\text { solution }\end{array}$ & $\checkmark$ & $\checkmark$ & $\begin{array}{l}\text { Discovering further aster- } \\
\text { oids }\end{array}$ & $\begin{array}{l}\text { Digital tracking tech- } \\
\text { nique revealed } 1 \text { first } \\
\text { asteroid }\end{array}$ \\
\hline [10] & $\begin{array}{l}\text { Distributed } \\
\text { cloud } \\
\text { solution }\end{array}$ & $\sqrt{ }$ & $\checkmark$ & $\begin{array}{l}\text { Discovering further fainter } \\
\text { asteroids }\end{array}$ & $\begin{array}{l}\text { able to detect aster- } \\
\text { oids down to about } 21 \\
\text { mag }\end{array}$ \\
\hline [11] & $\begin{array}{l}\text { Distributed } \\
\text { cloud } \\
\text { solution }\end{array}$ & $\sqrt{ }$ & $\sqrt{ }$ & $\begin{array}{l}\text { Discovering up to } 180 \\
\text { fainter asteroids }\end{array}$ & $\begin{array}{ll}256 & \text { asteroids } \\
\text { discovered } & \text { were } \\
\end{array}$ \\
\hline [12] & $\begin{array}{l}\text { Distributed } \\
\text { cloud } \\
\text { solution }\end{array}$ & $\checkmark$ & $\checkmark$ & $\begin{array}{l}\text { Discovering more fainter } \\
\text { asteroids }\end{array}$ & $\begin{array}{l}\text { discovered } 200 \text { fainter } \\
\text { asteroids }\end{array}$ \\
\hline [13] & $\begin{array}{l}\text { Distributed } \\
\text { cloud } \\
\text { solution }\end{array}$ & $\sqrt{ }$ & $\checkmark$ & $\begin{array}{l}\text { Hough } r \begin{array}{r}\text { transformation } \\
\text { detects }\end{array} \text { asteroids } \\
\text { and makes real-time } \\
\text { updates/results on data. }\end{array}$ & None \\
\hline [14] & $\begin{array}{l}\text { Distributed } \\
\text { cloud } \\
\text { solution }\end{array}$ & $\checkmark$ & $\sqrt{ }$ & $\begin{array}{l}\text { automatic image reduc- } \\
\text { tion, detection and valida- } \\
\text { tion of moving objects }\end{array}$ & $\begin{array}{l}\text { detects more asteroids } \\
\text { (by } 8-41 \% \text { ) than other } \\
\text { tools }\end{array}$ \\
\hline
\end{tabular}

For an easier image manipulation the tool SAO DS9 offers an astronomical image viewer that operates on a FITS image to automatically detect objects [20].

For a real-time, 3D, astronomy visualisation software, based on chartig about 1 billion stars of our Galaxy in the Gaia group of the Astronomisches Rechen-Institut, the modern Gaia Sky tool is the perfect option [22].

SkyCat on one hand is a tool that combines visualization of images and access to catalogs and archive data for astronomy [23]. TopCat on the other hand is a tool for operations on catalogues and tables [26].

The Common Astronomy Software Applications package 
TABLE II: Available tools overview.

\begin{tabular}{|c|l|l|c|}
\hline Name & $\begin{array}{l}\text { Parallel / dis- } \\
\text { tributed }\end{array}$ & Operating systems & Latest release \\
\hline IRAF [15] & No & Linux, Windows & 2012 \\
\hline PyRAF [16] & No & Linux & 2015 \\
\hline PyCDA [17] & No & Linux & 2018 \\
\hline SExtractor [18] & No & Linux & 1995 \\
\hline TERAPIX [19] & No & Linux & 2016 \\
\hline SAO Ds9 [20] & No & Linux & 2019 \\
\hline GAIA [21] & No & Linux, Windows, MacOS & 2018 \\
\hline Gaia Sky [22] & No & Linux, Windows, MacOS & 2013 \\
\hline SkyCat [23] & No & MacOS, Linux & 2019 \\
\hline CASA [24] & No & MacOS,Linux & 2019 \\
\hline World Wide Telescope [25] & No & Windows, MacOS, Linux & 2016 \\
\hline TopCat [26] & No & Windows, Linux & 2019 \\
\hline GalayZoo [27] & No & Linux & 2009 \\
\hline Seti@Home [28] & Yes & MacOS, Linux, Windows & 2011 \\
\hline IDATA [29] & No & Linux & 2018 \\
\hline Astrometrica [30] & No & Windows & 2018 \\
\hline NEARBY [14] [31] & Yes & Linux, Docker & 2019 \\
\hline EXTRACTOR [32] & No & Windows, MacOS, Linux & 2018 \\
\hline IMG [33] & No & Windows, MacOS, Linux & 2018 \\
\hline PISA [34] & No & Windows, MacOS, Linux & 2018 \\
\hline SPLAT [35] & No & Windows, MacOS, Linux & 2018 \\
\hline SST [36] & No & Windows, MacOS, Linux & 2018 \\
\hline Sky Tools 4 Imaging [37] & No & Windows, MacOS & 2018 \\
\hline Sky Tools 4 Visual [37] & No & Windows, MacOS & 2018 \\
\hline & & & \\
\hline & & \\
\hline
\end{tabular}

(CASA) is a tool developed with the primary goal of supporting the data post-processing needs of the next generation of radio astronomical telescopes (such as ALMA and VLA)[24].

The World Wide Telescope represents a powerful and free visualization program that gives you instant access to the most complete collection of astronomical imagery available [25].

EXTRACTOR is a tool based on SExtractor, for automatically detecting objects on an astronomical image and building a catalogue of their properties. Is particularly suited for the reduction of large scale galaxy-survey data, but also performs well on other astronomical images, accepting more formats than SExtractor [32].

Simple Image Data Access or IMG is a library that can be used together with different tools for simpler image detection [33].

The Position Intensity and Shape Analysis group or PISA was specially created for automatically detecting and measuring objects on an astronomical images, providing the ability to display the shapes and positions of the detected objects over the image and convert its results into catalogues [34].

The Spectral Analysis Tool (SPLAT) represents a graphical tool for displaying, comparing, modifying and analysing astronomical spectra stored in NDF, FITS and TEXT files as well 
as the new NDX format. SPLAT is now part of the STARJAVA collection) [35].

The Simple Software Tools (SST) is actually a port of the simple software tools package is available for UNIX platforms. This is now part of the EAO Starlink release[36].

The Traitement Elementaire, Reduction et Analyse des PIXels (TERAPIX) is an astronomical data reduction centre dedicated to the processing of very large data flows from digital sky surveys (like the CFHTLS, WIRDS or WUDS, NGVS, CFHQSIR, KIDS/VIKING, UltraVISTA) and giant panoramic visible and near infrared cameras (like MegaCam and WIRCam at CFHT, or OmegaCam on the VST and VIRCam on VISTA at ESO/Paranal) [19].

The Sky Tool 4 Images replaces guesswork with accurate calculations. It can accurately estimate the signal to noise ratio under any conditions, allowing for much higher quality planning. Currently, imagers use experience, rules of thumb and generalizations to decide what to image, when to image, how long to expose in total, and what sub exposure times to use) [37].

The SkyTools 4 for Visual Observing [37] is somewhat similar to Sky Tool 4 Images.

The Galaxy Zoo is a tool used for finding galaxies and classificate them [27].

The Search for Extraterrestrial Intelligence (SETI@HOME) is a scientific tool whose goal is to detect intelligent life outside Earth. One approach, known as radio SETI, uses radio telescopes to listen for narrow-bandwidth radio signals from space. Such signals are not known to occur naturally, so a detection would provide evidence of extraterrestrial technology [28].

IDATA is a three year project funded by the National Science Foundation to engage a group of high school age students, their teachers, professional astronomers, software engineers, and design experts in the user-centered design process of data analysis software for astronomy [29].

Astrometrica is an interactive Windows software for scientific grade astrometric data reduction of CCD images, focusing on the validation and measurement of the minor bodies of the solar system (asteroids, comets, dwarf planets) [30].

To ease the understanding of the reader regarding these tools and to point the focus on the results and timing, a table would be suited. Further information regarding the existing tools and their optimization power (parallel or not) will be provided in Table II below.

\section{Proposed ARchitecture}

In this section we detail the proposed distributed cloud architecture meant to speedup the sequential code execution.

The generic sequential algorithm for digital tracking is presented as follows:

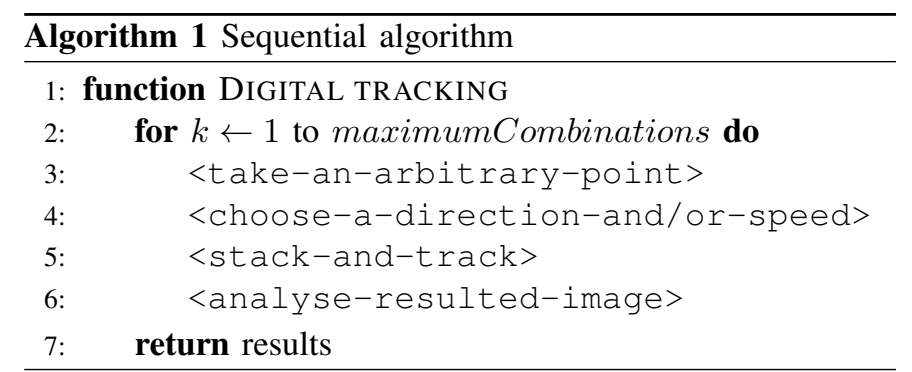

It can be seen that each loop can be trivially parallelized as there are no dependencies between iterations.

The proposed architecture envisioned two techniques: asteroid pairing and digital tracking.

The first aspect refers to pairing over 800,000 existing orbits with more than 1 million trajectories of celestial objects (as per ONS/MPC) observed during a single night only, in an attempt to identify existing NEAs.

The second objective refers to identifying and tracking asteroids that move rapidly in the night sky. The procedure refers to a stacking and shifting model for images in all assumed trajectory directions for identifying new and existing faint asteroids. The large amount of possible combinations (direction and speed) is suited for a cloud platform for CPUGPU image processing that elastically scales based on the number of real-time images.

While parallelism in the first case involves computing each field on a different CPU, the second case is more interesting: for each direction allocate a multi-core processor, and run in parallel for each direction (per CPU) several angular speeds (per core) (cf. Figure 5). Present day cloud platforms allow us to rent at reasonable prices virtual machines with up to 96 virtual CPUs and thousands of GPU cores suited for multiand many- core computations. These machines are easily accessible and configurable with MPI, OpenMP and CUDA.

A global picture of the sequential and proposed cloud enabled architecture is presented in Figures 6 and 7. A typical workflow would proceed as follows: A user would login to the web Ui interface to access the system. The data would be sent to a script that will handle the initialization and boot of the VMs as well as data distribution. Each VM will run its own tools (e.g., IRAF and SExtractor) and will communicate with external astronomical services (database queries) for data processing and analysis. Results will be stored in a cloud database. Further information about the specific steps are detailed below. It can be seen that both the sequential and the parallel versions run on the VMs. The only difference being the way data is processed and analyzed.

As already mentioned in Section I both digital tracking and asteroid pairing have joint modules used mostly for image clean-up, stack-and-track, and database query:

1) Aligning images: finding the spatial mapping, for example elements in one image into meaningful correspondence with elements in a second image;

2) Cleaning image noise 


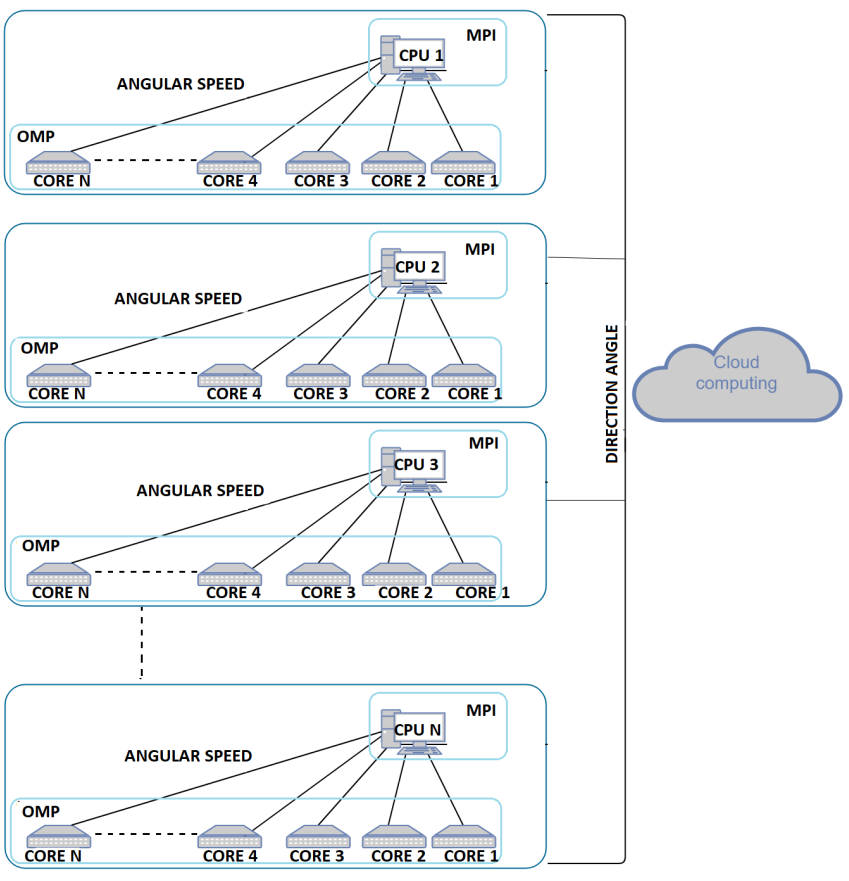

Fig. 5: Proposed parallel approach for digital tracking.

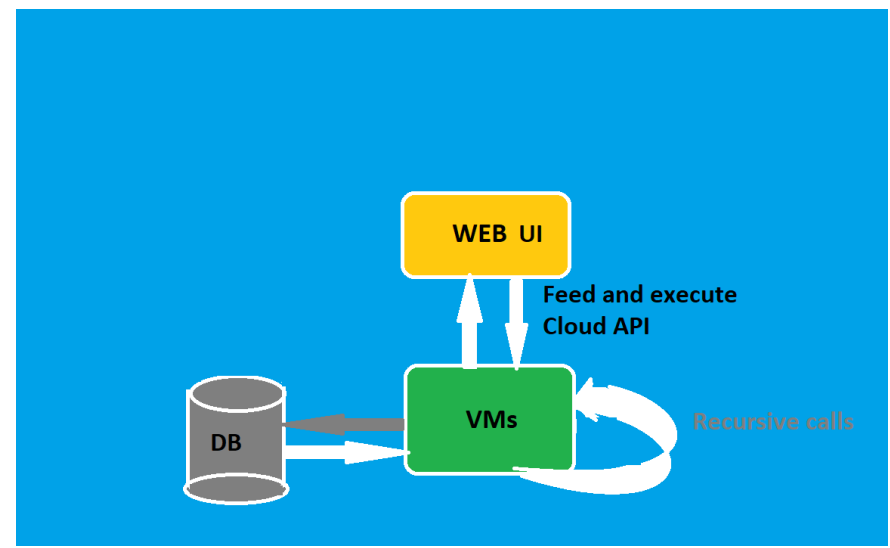

Fig. 6: Sequential architecture.

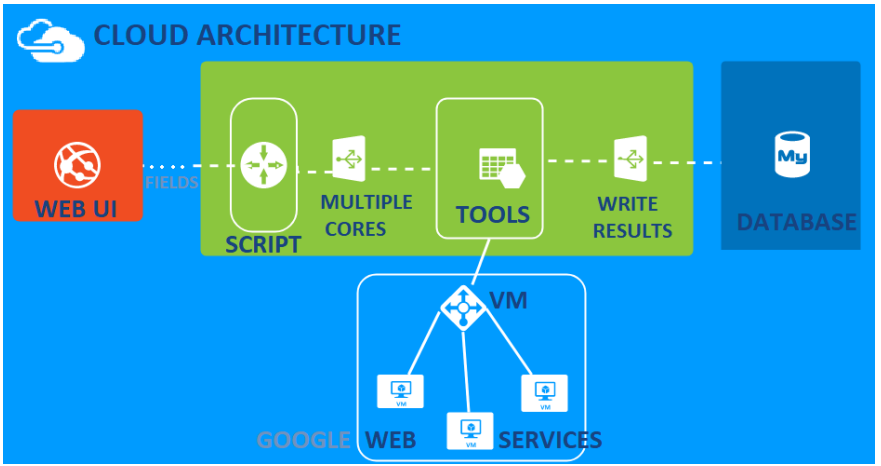

Fig. 7: Proposed cloud architecture. a) Image Pre-processing: correcting artifacts (bias, flat field, bad pixels, vignetting) from raw imput;

i) Bias correction: thermic internal noise of the camera;

ii) Flat field correction: stain optical correction of the instrume;

iii) Bad pixel correction;

iv) Vignetting: shadows in the camera.

b) Field correction: corrects the field distortions and resamples the raw images;

i) Object detection: (SExtractor) identifying all the objects (starts, galaxies,etc) in the image);

ii) Field recognition: (Scamp) identifies stars in images;

iii) Image re-construction: pixel by pixel for a sharp image.

This step can parallelized by distributing it on multiple CPU/cores. Specifically, each field can be processed independently and in parallel.

\section{Digital tracking algorithm}

The next step will be the actual stack-and-track (see Algorithm 1):

Stack and track images on a direction and/or a speed (see Figure 4) refers to stacking images obtained after shifting in two dimensions (by direction and angular speed - both assumed). It proceeds as follows:

First, we choose an arbitrary point or image in the sky which will be subject to parallelizing since a new object will be chosen every time the parameters change (direction and/or speed).

In case an object is being retrieved, the identified trajectory will be compared against the existing database (with almost one million known trajectories), resulting a newly identified object or recovery of an old one.

If the object is not retrieved the arbitrary point will be searched in another direction and the above procedure is reapplied. To speedup the search, the procedure will be applied in parallel without waiting for found/not found results of each scenario.

\section{Asteroid pairing algorithm}

Since every night MPC (Minor Planet Center) receives captures of over one million unidentified objects, every single object could be paired against the over 800,000 known trajectories in order to improve an already discovered one or for discovering a new one:

$$
\begin{aligned}
& 800,000 \text { trajectories } \\
& \quad \times 1,000,000 \text { unknown objs } \\
& \quad=800,000,000,000 \text { combinations }
\end{aligned}
$$

These growing numbers are being evaluated for the moment by a team of only few people at the MPC that have increasiung difficulties to pair such a growing amount of data sequentially. 
This is why a tool with a parallel architecture is required to evaluate the stored Big Data in order to obtain a real time results.

After the image alignment and cleanup the algorithm proceeds as follows:

The resulted images are stacked and objects are identified by identifying star like objects from linear ones. In this approach asteroids appear as points while stars move.

The candidate asteroid will be paired against the known trajectories, if it is found among them, the orbit refinement will be made, if not a new discovery (NEA) will be registered and the search will continue on the next object. Given that multiple fields can be studied per night (upto 100) each field can be analyzed in parallel.

\section{Discussion and Future Work}

The speed, reliability, performance and cross-compatibility are of paramount importance, this what the proposed distributed architecture proposes to archive. Over the next couple of years we will implement and demonstrate the explained architecture. Using distribution and parallelism, we will help expanding the tool options of researchers when dealing with Big Data generated by sky analyzing. (cf. Figure 7)

\section{CONCLUSION}

According to [7] the need for developing new image processing architectures is given by the increasing amount of survey and faster processing.

As per Table II we have seen that there are only very few tools trying to cover some small parts of the vast sky analyze and even fewer that are actually designed for distributed execution.

This is why a distributed architecture is needed to provide a comprehensive set of approaches that support detection of faint NEAs in near real-time, reducing the effort of scientific teams, using the new digital tracking technique.

This kind of architecture offers a powerful solution to common pitfalls of image analysis such as visual correlation and correction and offers a distributed parallel solution to reduce the processing pairing time.

Future steps will consist of implementing, testing, and validating the proposed architecture in CPU and GPU environments, using real observing images provided by the EURONEAR project.

\section{REFERENCES}

[1] W. Bottke et al., Asteroids III. British Library, 2002.

[2] A. N. Heinze et al., "Digital tracking observations can discover asteroids ten times fainter than conventional searches," 2015. [Online]. Available: https://arxiv.org/pdf/1508.01599.pdf

[3] "Euronear," 2006. [Online]. Available: http://www.euronear.org/

[4] L. Benacchio, "Retrieval of astronomical information from padovaasiago observatory plates archives," International Astronomical Union Colloquium, pp. 233-236, 1982.

[5] A. Boattini, G. D. Abramo, G. Forti, and R. Gal, "The arcetri neo precovery program," Astronomy \& Astrophysics, pp. 293-307, 2001.

[6] O. Vaduvescu et al., "Euronear: Data mining of asteroids and near earth asteroids," Astronomical Notes/Astronomische Nachrichten, vol. 330, no. 7, 2009.
[7] _ "Mega-precovery and data mining of near-earth asteroids and other solar system objects," ACM 2014, vol. 508, no. 425P, 2014.

[8] — "Data mining of near earth asteroids in the subaru suprime-cam archive," Astronomische Nachrichten, vol. 338, no. 5, pp. 527-535, 2017.

[9] C. Zhai et al., "Detection of faint fast-moving near-earth asteroid using the synthetic tracking technique," The Astrophysical Journal, vol. 792, no. 1,2014

[10] T. Yanagisawa et al., "Automatic detection algorithm for small moving objects," Publications of the Astronomical Society of Japan, vol. 57 no. 2, p. 399408, 2005.

[11] M. Shao et al., "Finding very small asteroid using synthetic tracking," The Astrophysical Journal, 2014.

[12] A. Heinze et al., "Digital tracking observations can discover asteroids ten times fainter than conventional searches," The Astrophysical Journal, 2015.

[13] H. Chi-Sheng et al., "The distributed asteroid discovery system for large astronomical data," Journal of Network and Computer Applications, vol. 93.

[14] "Ready for euronear nea surveys using the nearby moving source detection platform," 2019. [Online]. Available: http://www.euronear.org/publications/Vaduvescu_Paper115.pdf

[15] "Iraf," 2012. [Online]. Available: http://ast.noao.edu/data/software

[16] "Pyraf," $2015 . \quad$ [Online]. Available: http://www.stsci.edu/institute/software_hardware/pyraf

[17] "Pycda: An open-source library for automated crater detection," 2018. [Online]. Available: http://planetarycraterconsortium.nau.edu/KlearPCC9.pdf

[18] "Sextractor: Software for source extraction," 1995. [Online]. Available: https://aas.aanda.org/articles/aas/pdf/1996/08/ds1060.pdf

[19] "Terapix," 2012. [Online]. Available: http://terapix.calet.org/terapix.iap.fr/

[20] “ds9,” 2019. [Online]. Available: http://ds9.si.edu/site/Home.html

[21] "Gaia," 2003. [Online]. Available: http://starwww.dur.ac.uk/ pdraper/gaia/gaia.html

[22] "Gaia sky," 2019. [Online]. Available: https://zah.uniheidelberg.de/institutes/ari/gaia/outreach/gaiasky/

[23] "Skycat," 2011. [Online]. http://www.eso.org/sci/observing/tools/skycat.html

[24] "Common astronomy software applications package," 2018. [Online]. Available: https://casa.nrao.edu/

[25] "Common astronomy software applications package," 2010. [Online]. Available: https://wwtambassadors.org/

[26] "Topcat," 2003. [Online]. Available: http://www.star.bris.ac.uk/ mbt/topcat/

[27] “Galaxy zoo," 2007. [Online]. Available: https://data.galaxyzoo.org/

[28] "Seti@home," $2007 . \quad$ [Online]. Available: https://setiathome.berkeley.edu/sah_about.php

[29] "Idata," $2018 . \quad$ [Online]. Available: https://www.glaseducation.org/idata.html

[30] "Astrometrica," 2001. [Online]. Available: http://www.astrometrica.at/

[31] "Nearby platform for automatic asteroid detection and euronear surveys," 2019. [Online]. Available: http://www.euronear.org/publications/NEARBY-ESA-NEOSST1Paper.pdf

[32] "Extractor," 2013. [Online]. Available: http://starwww.dur.ac.uk/ pdraper/extractor/extractor.html

[33] "Img," 2013. [Online]. Available: http://starwww.dur.ac.uk/ pdraper/img/img.html

[34] "Pisa," 2013. [Online]. Available: http://starwww.dur.ac.uk/ pdraper/pisa/pisa.html

[35] "Splat," 2013. [Online]. www.dur.ac.uk/ pdraper/splat/splat.html

[36] "Sst," 2013. [Online]. www.dur.ac.uk/ pdraper/sst/sst.html

[37] "Sky tool 4," 2018. https://www.skyhound.com/index.html
Available: http://star-

Available: http://star-

[Online]. Available: 